\title{
The effects of lumbar sympathectomy on bone and soft tissue haemodynamics of the leg recorded using near infrared spectroscopy: A case report
}

\author{
Robert M Meertens, Karen M Knapp, Francesco Casanova, William D Strain \\ Medical Imaging, University of Exeter, Exeter, United Kingdom
}

Received: September 16, 2016

DOI: $10.5430 /$ jbei.v3n1p28
Accepted: October 30, $2016 \quad$ Online Published: November 3, 2016

URL: http://dx.doi.org/10.5430/jbei.v3n1p28

\begin{abstract}
Near infrared spectroscopy (NIRS) is an established tool for the measurement of microvascular haemodynamics in different tissue types. This case report outlines the novel use of NIRS for measuring total oxygenation index (TOI) and relative oxygenated and deoxygenated haemoglobin concentration changes as markers of oxygenation and blood volume in muscle and bone tissue. In particular, NIRS is used to examine differences between the left and right leg in a participant who has experienced a permanent unilateral lumbar sympathectomy following an anterior lumbar intervertebral fusion. Anatomical sites at the lateral head of the gastrocnemius, tibial diaphysis and proximal tibia were investigated with NIRS during an arterial occlusion protocol at the distal femur. Consistent differences were observed between the sympathectomised left leg and the normal right leg. These included reduced baseline TOI, reduced deoxygenation rates during occlusion, and reduced reoxygenation rates post occlusion release in the sympathectomised leg at all anatomical sites. This case report demonstrates the potential of NIRS as a research tool for investigating the microvascular effects of lumbar sympathectomy. This may be useful for further investigation into the merit of chemical lumbar sympathectomy for the treatment of a variety of conditions, including hyperhidrosis and peripheral vascular disease.
\end{abstract}

Key Words: Near infrared spectroscopy, Sympathectomy, Bone, Muscle, Total oxygenation index

\section{INTRODUCTION}

Lumbar sympathectomy is an established interventional technique that results in inhibition of sympathetic regulation of the lower legs. This can have clinical benefits, as reduced sympathetic response can lead to chronic vasodilation, pain suppression, and reduced sweating. ${ }^{[1]}$ However, it is generally accepted that the physiological mechanisms behind these effects are not completely understood. ${ }^{[2]}$ Nevertheless, lumbar sympathectomy has been used as a treatment option for conditions such as peripheral vascular disease, Raynaud's disease and hyperhidrosis. ${ }^{[1,3,4]}$

Lumbar sympathectomy can be achieved by surgical resection or clamping of sympathetic ganglion; or by chemical treatment, typically with phenol. Chemical lumbar sympathectomy (CLS) is usually performed under less invasive image guided injections. ${ }^{[2]}$ Lumbar sympathectomy can also be a temporary or permanent side effect of lumbar surgery caused by resultant damage to the nearby lumbar sympathetic ganglion. Hrabalek et al., 2015 notes a wide range of reported incidence of sympathetic dysfunction following

\footnotetext{
*Correspondence: Robert M Meertens; Email: r.m.meertens@exeter.ac.uk; Address: South Cloisters Building, St Luke’s Campus, University of Exeter, Heavitree Road, Exeter, EX1 2LU, United Kingdom.
} 
anterior lumbar interbody fusions (ALIF), with incidence ranging between $1 \%$ and $43 \%$ of ALIF cases. ${ }^{[5]}$

\section{CASe PResentation}

This case report investigates a rare case of permanent unilateral lumbar sympathectomy. The participant was a 41 year old female with a BMI of 28.1. As a result of previous spinal surgery 22 years previously following a chance fracture of L2, an anterior spinal fusion of L2 and L3 was performed using fixation of the vertebral bodies (see Figure 1). A posterior fusion was not considered appropriate due to the involvement of the pedicles in the fracture and therefore the anterior fusion was conducted, with removal of the left 12 th rib, which was utilized as a bone graft between L2 and L3.

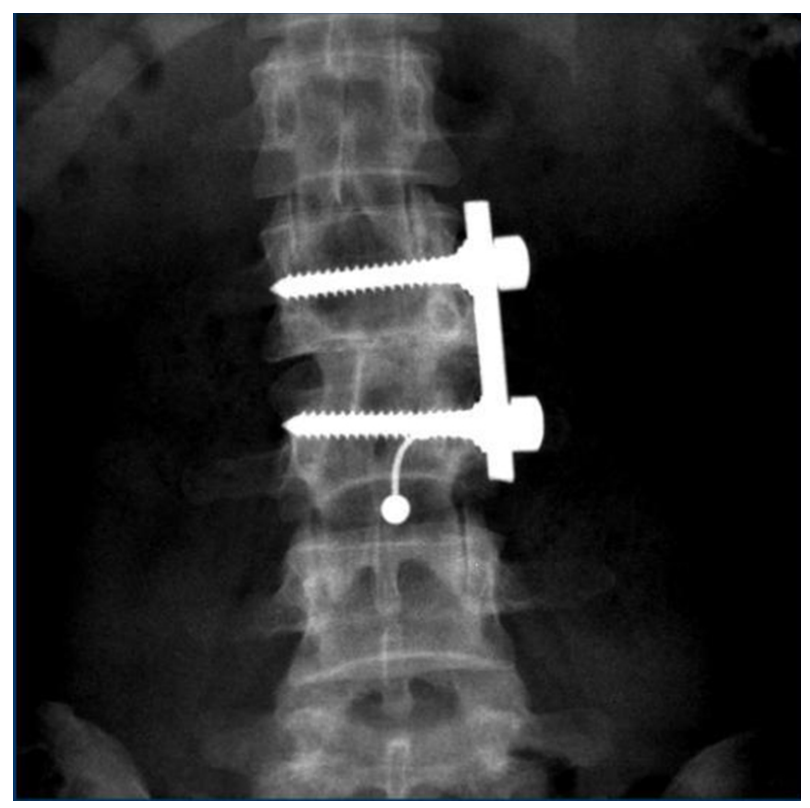

Figure 1. Anterior Lumbar Intervertebral Fusion (ALIF) of the participant at L2/L3 level

Note. Navel ring artefact present

The participant recovered well, with good function and movement, though was left with a unilateral lumbar sympathectomy of the left leg. The participant has a permanent "hot foot" with a 4.0 degree Celsius temperature difference between the participant's left and right foot found at baseline, a sign of permanent sympathectomisation. ${ }^{[6]}$ The participant was normotensive and an otherwise active and healthy non-smoker. The participant was relatively active, undertaking a minimum of 4.5 hours of moderate and high intensity exercise per week including team sports and strength and resistance training. The participant was opportunistically recruited by the primary author to investigate any resulting haemodynamic differences in bone tissue and soft tissue Published by Sciedu Press between the normal and sympathectomised lower legs.

\subsection{Methods}

This study was performed under institutional ethics approval with the informed consent of the participant. The Hamamatsu NIRO-200NX near infrared spectroscopy system was used to calculate absolute tissue oxygenation (TOI), oxygenated haemoglobin concentration $(\mathrm{O} 2 \mathrm{Hb})$, deoxygenated haemoglobin concentration $(\mathrm{HHb})$ and a normalized total tissue haemoglobin index by spatially resolved spectroscopy (SRS) at one second intervals. This system uses low power pulsed laser light sources at $735 \mathrm{~nm}, 810 \mathrm{~nm}$ and $850 \mathrm{~nm}$ to interrogate a tissue sample volume to a depth of $\sim 2 \mathrm{~cm}$. Reflectance spectroscopy is routinely used to assess skeletal muscle oxygenation ${ }^{[7]}$ and has a clinical application in monitoring cerebral oxygenation. ${ }^{[8,9]}$

The participant was asked to rest supine for 15 minutes to achieve a relaxed baseline state. Arterial saturation and pulse were monitored throughout the testing duration for any obvious systematic changes during testing.

Two superficial bony sites were measured on each leg individually. Firstly, the medial surface of the tibial diaphysis (TD) at a point mid-way between the palpatable tibial tuberosity and medial malleolus; and secondly on the medial surface of the proximal tibia (TP) $2-4 \mathrm{~cm}$ below the palpatable tibial tuberosity. For each of these four bony sites, baseline oxygenation was recorded as a 20 seconds mean prior to an arterial occlusion protocol. Here a blood pressure cuff placed around the distal femur was rapidly inflated to a suprasystolic $200 \mathrm{mmHg}$ for four minutes. The occlusion was then released with further monitoring until baseline was restored. An additional five minutes was allowed for baseline restoration prior to testing the next anatomical location with another arterial occlusion protocol. During all arterial occlusions, simultaneous soft tissue measurements were taken at the area of the lateral head of the gastrocnemius muscle using a $3 \mathrm{~cm}$ source/detector spacing, measuring a tissue depth of $\sim 1.5 \mathrm{~cm}$.

\subsection{Results}

Comparisons of bone and soft tissue data in both the left and right leg of the patient generally showed consistent trends. The sympathectomised left leg had a lower baseline oxygenation for the proximal tibia $(81.58 \%[+/-0.13 \%]$ vs. $85.58 \%$ [+/-0.30\%]) and tibial diaphysis sites $(82.75 \%$ [+/-0.24\%] vs. $83.48 \%$ [+/-0.33\%]) as well as for the corresponding soft tissue readings $(75.06 \%[+/-0.05 \%]$ vs. $76.56 \%[+/-0.08 \%])$ and $74.97 \%(+/-0.10 \%)$ vs. $77.88 \%(+/-0.06 \%)$. Upon arterial occlusion at all four sites the sympathectomised left leg had a slower rate of deoxygenation, suggesting lower oxygen 
consumption metabolism for the proximal tibia and tibial diaphysis sites (as well as corresponding soft tissue readings) in the sympathectomised leg. Likewise, the reperfusion reoxygenation rates were slower in the sympathectomised left leg across all bone and soft tissue sites upon release of the arterial occlusion (see Table 1 and Figure 2 for results).

Table 1. Baseline TOI, desaturation rates and resaturation rates during arterial occlusion testing

\begin{tabular}{llll}
\hline & $\begin{array}{l}\text { Baseline } \\
\text { Oxygenation } \\
\text { (TOI \% +/-SD\%) }\end{array}$ & $\begin{array}{l}\text { TOI } \\
\text { Desaturation } \\
\text { Rate (\%/s) }\end{array}$ & $\begin{array}{l}\text { TOI } \\
\text { Resaturation } \\
\text { Rate (\%/s) }\end{array}$ \\
\hline Right TD & $83.48(+/-0.33)$ & -0.0405 & 0.221 \\
Left TD & $82.75(+/-0.24)$ & -0.0292 & 0.140 \\
Right TP & $85.58(+/-0.30)$ & -0.0220 & 0.359 \\
Left TP & $81.58(+/-0.13)$ & -0.0166 & 0.034 \\
Right Muscle with TD & $77.88(+/-0.06)$ & -0.0448 & 0.621 \\
Left Muscle with TD & $74.97(+/-0.10)$ & -0.0231 & 0.523 \\
Right Muscle with TP & $76.56(+/-0.08)$ & -0.0487 & 0.757 \\
Left Muscle with TP & $75.06(+/-0.05)$ & -0.0255 & 0.493 \\
\hline
\end{tabular}

Total oxygen haemoglobin concentration (nTHI) remained within $+/-10 \%$ during the arterial occlusions with the corre- sponding mirrored pattern of oxygenated and deoxygenated haemoglobin changes suggesting effective arterial occlusions, as expected with cuff pressures of a minimum $200 \mathrm{mmHg}$. There were no significant changes in arterial oxygenation saturations or pulse rate during testing.

\section{Discussion}

CLS remains an existing treatment option for a number of clinical indications. ${ }^{[1,3,4]}$ It is believed the clinical benefits of pain relief and increases in localised perfusion are mainly due in part to increased vasodilation in the absence of sympathetic tone. However, NICE guidelines do not currently recommend the use of CLS on the basis of a lack of proven benefit and a weak understanding on the mechanisms of action behind lumbar sympathectomy. ${ }^{[2]}$

It is well documented that with the expected vasodilation associated with CLS, increased temperature and blood perfusion in the cutaneous tissue of the sympathectomised area will result. ${ }^{[4,6]}$ Improvements in skin perfusion have been demonstrated using optical techniques such as laser Doppler flowmetry, although changes in blood volume cannot be measured using these techniques. ${ }^{[4,10]}$

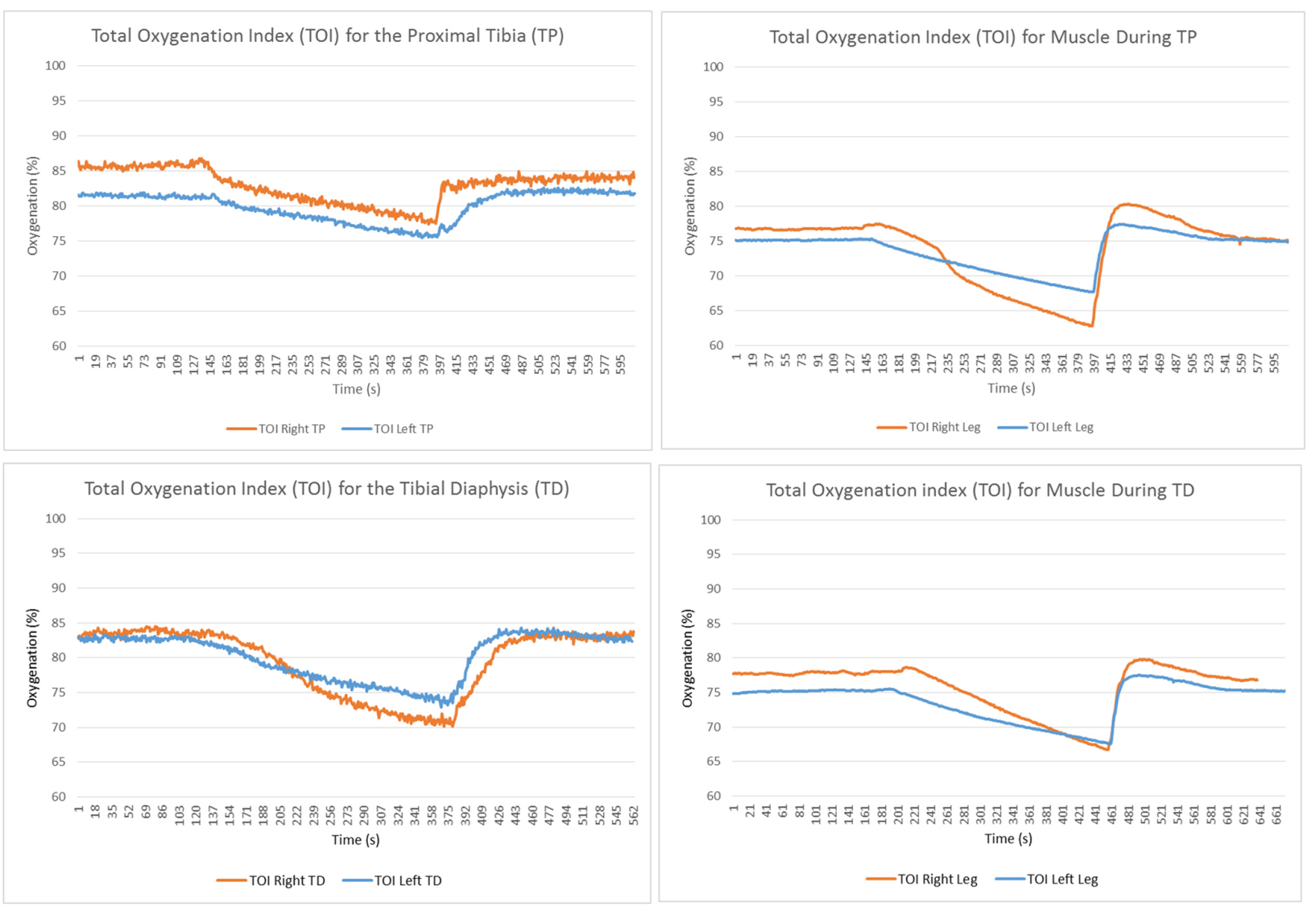

Figure 2. Total Oxygenation Indices for (a) the Proximal Tibia (TP)(upper left); (b) associated muscle readings(upper right); (c) the Tibial Diaphysis (TD)(lower left); (d) the associated muscle readings during the testing period(lower right) 
However, there is generally a paucity of data on the effects of sympathectomy on deeper tissues, such as skeletal muscle, and particularly in-vivo studies of human bone. Sympathetic and thermoregulatory mechanisms have been demonstrated in human muscle tissue where vasodilation of active skeletal muscle can be simultaneously counterbalanced by vasoconstriction in inactive muscles and organs. ${ }^{[11]}$ This has been studied using laser Doppler Flowmetry, although this technique is generally limited by penetration depth when sampling deeper tissues. ${ }^{[12]}$ Therefore many studies on the effectiveness of CLS rely on pragmatic markers such as reduction in pain scores or rate of ulcer healing.

Both sympathetic and sensory nerve fibres exist in bone tissue and course through Volkmann's and Haversian canal systems, influencing vasoconstriction in cortical bone. It is also recognized that the sympathetic nervous system has a role to play in regulating bone metabolism. In murine models, bone loss has been observed post sympathectomy. ${ }^{[13]}$ Likewise in induced cases of spinal cord injury in murine models, denervation was linked with changes to vasoregulation which affected bone metabolism, and bone loss, more than disuse mechanisms alone. Similarly the observed increase in pain, bone loss and increased sympathetic activation in complex regional pain syndrome indicates an important role of the sympathetic nervous system in bone tissue. ${ }^{[13]}$

This case study presents a novel way of potentially measuring haemodynamic changes in bone tissue in response to sympathectomisation using NIRS. It can be seen from results that when sampling both muscle and bone sites, NIRS produced consistent trends between the sympathectomised and unaffected leg across measurement sites. NIRS already has some emerging precedence for use in haemodynamic measurements of bone tissue. ${ }^{[14]}$ As this is a new application of NIRS testing, further use of this technique would be required to elucidate the explanatory mechanisms behind results in broader populations.

In this case report it can be seen that the rate of oxygenation decrease upon arterial occlusion (a marker of oxygen consumption metabolism) was decreased in the sympathectomised leg. Oxygen extraction is a measure of the rate of oxygen uptake as a proportion of the concentration of available blood in the tissue and therefore if there is more blood volume in the left sympathectomised leg then the rate of extraction may be lower in this leg, as observed.

The reoxygenation rate post occlusion release (a marker of blood perfusion as arterial blood re-enters tissue) was also reduced in the sympathectomised left leg. Here with faster tissue reperfusion post occlusion release there would be ex- pected to be more efficient oxygen transfer to tissue. With a potentially larger blood volume and vasodilation in the sympathectomised leg prior to occlusion release there may be reduced intra-arterial pressure and therefore reduced reperfusion rates.

It was surprising that our results do not reflect vasodilation in the absence of sympathetic tone in the sympathectomised left leg leading to an increased baseline oxygenation in skeletal muscle and bone. One potential theory is that chronic vasodilation in the leg has led to a larger blood volume including more venous pooling. This relative increase in deoxygenated haemoglobin means NIRS readings would report a lower oxygenation percentage.

However all of these observations can only be theorized in the situation of a case study and without an existing evidence base in human bone tissue for comparison. Furthermore, in this case study, unilateral sympathectomy has resulted as a complication from lumbar surgery, which is a potential limitation, as results could differ from CLS. For example, the preceding surgery was carried out 22 years ago and the body may have adapted to chronic changes, which may differ from the short term benefits predominantly reported in the literature around CLS.

\section{Conclusion}

Although data are isolated to one participant, the results from this case study appeared to be consistent in our small sample of measurements across both legs at two bone sites, and repeated measurements at one muscle site. NIRS is now a recognized research technique for measuring tissue oxygenation and haemoglobin concentration in both muscle and bone tissue. ${ }^{[12,14]}$ Differences between the right and left leg were consistent and therefore support the suggested potential role of the sympathetic nervous system in regulation of bone and muscle haemodynamics. The use of NIRS as an investigative technique may therefore be useful for further investigation in larger cohorts of those with lumbar sympathectomy in order to gain more physiological data on the effect of sympathetic innervation on muscle and bone tissue. A better physiological understanding of the relationship of sympathetic innervation in bone and muscle microvasculature could help guide further treatment options using CLS.

\section{ACKNOWLEDGEMENTS}

Acknowledgements go to Clare Thorn for her help with this research. Also to the College of Radiographers Industrial Partnership Scheme (CoRIPS) for their financial support of this research programme via the CoRIPS Doctoral Fellowship Grant. 


\section{REFERENCES}

[1] Nesargikar PN, Ajit MK, Eyers PS, et al. Lumbar chemical sympathectomy in peripheral vascular disease: does it still have a role? International journal of surgery (London, England). 2009; 7(2): 145 9. PMid:19237331. http://dx.doi.org/10.1016/j.ijsu. 20 09.01 .004

[2] National Institute of Health and Care Excellence. Peripheral arterial disease: diagnosis and management (CG147). 2012.

[3] Koman LA, Smith BP, Pollock FE, et al. The microcirculatory effects of peripheral sympathectomy. The Journal of Hand Surgery. 1995; 20(5): 709-17. http://dx.doi.org/10.1016/S0363-5023(05 ) 80419-8

[4] Kao MC, Tsai JC, Lai DM, et al. Autonomic activities in hyperhidrosis patients before, during, and after endoscopic laser sympathectomy. Neurosurgery. 1994; 34(2): 262-8; discussion 8.

[5] Hrabalek L, Sternbersky J, Adamus M. Risk of sympathectomy after anterior and lateral lumbar interbody fusion procedures. Biomedical papers of the Medical Faculty of the University Palacky, Olomouc, Czechoslovakia. 2015; 159(2): 318-26. PMid:24263213.

[6] Butler-Manuel PA, Justins D, Heatley FW. Sympathetically mediated anterior knee pain. Scintigraphy and anesthetic blockade in 19 patients. Acta Orthop Scand. 1992; 63(1): 90-3. http://dx . doi .org /10.3109/17453679209154858

[7] Ferrari M, Muthalib M, Quaresima V. The use of near-infrared spectroscopy in understanding skeletal muscle physiology: recent developments. Philos Trans A Math Phys Eng Sci. 2011; 369(1955): 4577-90. PMid:22006907. http://dx.doi.org/10.1098/rsta. 2011.0230
[8] Leal-Noval SR, Cayuela A, Arellano-Orden V, et al. Invasive and noninvasive assessment of cerebral oxygenation in patients with severe traumatic brain injury. Intensive Care Med. 2010; 36(8): 1309-17. PMid:20502869. http://dx.doi.org/10.1007/s0013 4-010-1920-7

[9] Fedorow C, Grocott HP. Cerebral monitoring to optimize outcomes after cardiac surgery. Current opinion in anaesthesiology. 2010; 23(1): 89-94. PMid:19920759. http://dx.doi.org/10.1097/ACO.0b0 $13 \mathrm{e} 3283346 \mathrm{~d} 10$

[10] Lantsberg L, Goldman M. Lower limb sympathectomy assessed by laser Doppler blood flow and transcutaneous oxygen measurements. Journal of Medical Engineering \& Technology. 1990; 14(5): 182-3. http://dx.doi.org/10.3109/03091909009009956

[11] Korthuis RJ. Skeletal Muscle Circulation. San Rafael (CA), USA: Morgan \& Claypool Life Sciences; 2011.

[12] Binzoni T, Leung TS, Boggett D, et al. Non-invasive laser Doppler perfusion measurements of large tissue volumes and human skeletal muscle blood RMS velocity. Physics in Medicine and Biology. 2003; 48(15): 2527-49. http://dx.doi.org/10.1088/0031-9 $155 / 48 / 15 / 318$

[13] He JY, Jiang LS, Dai LY. The roles of the sympathetic nervous system in osteoporotic diseases: A review of experimental and clinical studies. Ageing Research Reviews. 2011; 10(2): 253-63. PMid:21262391. http://dx.doi.org/10.1016/j.arr.2011.01.002

[14] Binzoni T, Spinelli L. Near-infrared photons: a non-invasive probe for studying bone blood flow regulation in humans. Journal of Physiological Anthropology. 2015; 34(1): 1-6. PMid:25636731. 